\title{
ÉVALUATION DU BIEN-ÊTRE CHEZ LES CARNIVORES DE COMPAGNIE : CHIENS ET CHATS
}

\author{
WELFARE ASSESSMENT IN COMPANION ANIMALS: DOGS AND CATS
}

\author{
Par Caroline GILBERT ${ }^{1}$ et Emmanuelle TITEUX ${ }^{2}$
}

(Communication présentée le 5 novembre 2020, manuscrit accepté le 5 mai 2021)

\begin{abstract}
RÉSUMÉ
Le bien-être animal fait référence à un état mental subjectif, positif, lié à la perception que l'animal a de son milieu. Les outils d'évaluation du bien-être animal se doivent ainsi de mesurer et de quantifier objectivement les émotions négatives et positives, ainsi que l'état mental de l'animal. Ces outils et leurs indicateurs, validés et employés actuellement pour les animaux de rente et de loisir, restent étonnamment très peu développés pour les animaux de compagnie, chiens et chats. A travers différents exemples, nous proposerons des éléments d'appréciation du bien-être chez les chiens et chats, à court terme, lors de la pratique vétérinaire courante, mais aussi au plus long terme lors de l'évaluation de leur qualité de vie.
\end{abstract}

Mots-clés : Bien-être animal, indicateurs du bien-être animal, carnivores domestiques, chien, chat

\begin{abstract}
-ABSTRACT
Animal welfare refers to a subjective and positive mental state linked to the animal's perception of its environment. Therefore, animal welfare assessment tools must objectively measure and quantify negative and positive emotions, as well as the animal's mental state. These tools and their indicators, despite their validation and use for production and leisure animals, are surprisingly little developed for companion animals, dogs and cats. Through several examples, we describe different criteria enabling the assessment of the well-being of dogs and cats on a short-term basis, during routine veterinary practice, but also in the longer term when evaluating their quality of life.
\end{abstract}

Key-Words: Animal welfare, animal welfare indicators, dogs, cats

\section{INTRODUCTION}

Le terme bien-être apparaît en France dans les années 1980, en référence à la traduction européenne de l'anglais " welfare " (Veissier \& Boissy, 2009), ce terme, pour certains auteurs, recouvrant à la fois l'état de bien-être d'un individu et sa protection (la bientraitance). Actuellement, il est admis par les différents experts que la définition du bien-être animal s'applique aux animaux domestiques, de compagnie, de laboratoire ou aux animaux sauvages détenus en captivité, et ne s'applique donc pas aux animaux sauvages en liberté, qui ne dépendent pas de l'Homme (Mormede et al. 2018). Trois approches visant à définir le bien-être animal, complémentaires, ont émergé en fonction des années et des points de vue des différents scientifiques (Appleby, 1999) : les approches naturaliste, adaptative, et mentale (Veissier \& Boissy, 2009). Si les deux premières ont montré leurs limites (en effet, concernant nos chiens de compagnie, ceux-ci se sont totalement adaptés à leur milieu de vie actuel), la dernière, plus récente, place les émotions et les états mentaux au cœur de la définition du bien-être. En effet, d'après Duncan (2002), « le bien-être animal fait référence aux ressentis des animaux et non aux besoins

(1) DMV, PhD, Dipl. ECAWBM (AWSEL), Professeur en Ethologie fondamentale et appliquée à l'Ecole nationale vétérinaire d'Alfort, UMR 7179 CNRS/MNHN ; ENVA Bâtiment Camille Guérin, Ethologie, 7 avenue du Général de Gaulle 94700 Maisons-Alfort ; Laboratoire Mecadev, 1 avenue du petit château 91800 Brunoy, caroline.gilbert@vet-alfort.fr

(2) Emmanuelle Titeux, DMV, Dipl. ECAWBM (BM), Praticien hospitalier en médecine du comportement à l'Ecole nationale vétérinaire d'Alfort ; ENVA Bâtiment Camille Guérin, Éthologie, 7 avenue du Général de Gaulle 94700 Maisons-Alfort, etiteux@hotmail.com 
primaires ». Le bien-être peut alors être défini comme un état résultant de l'absence d'émotions négatives (peur, douleur, frustration), et de la présence d'émotions positives (joie, plaisir) (Fraser \& Duncan, 1998 ; Boissy et al. 2007a). Selon cette approche, le bien-être est un état subjectif de l'animal lié à sa perception de son milieu. Le groupe de travail « bien-être animal » de l'Anses propose ainsi une définition intégrant les différentes approches citées précédemment (Anses, 2018 ; Mormede et al. 2018) : « le bien-être d'un animal est l'état mental et physique positif lié à la satisfaction de ses besoins physiologiques et comportementaux, ainsi que de ses attentes. Cet état varie en fonction de la perception de la situation par l'animal ". Le concept de bien-être s'applique ainsi à la dimension mentale de la perception de l'animal en tant qu'individu dans son environnement. Cette dimension mentale porte l'attention sur le fait qu'une bonne santé, un niveau de production satisfaisant ou une absence de stress ne suffisent pas. Il faut aussi prendre en compte ce que l'animal ressent (Duncan, 1993), les perceptions subjectives déplaisantes, telles que la frustration, la douleur et la souffrance (Dawkins, 1988), mais aussi rechercher les signes d'expression d'émotions positives (Boissy et al. 2007b). Par ailleurs, il convient de prendre en compte les attentes de l'animal. Une attente est un processus mental généré par l'anticipation d'un évènement, auquel l'animal va se référer pour évaluer la valence de cet évènement, d'agréable à désagréable. Les attentes se traduisent par des réponses comportementales et physiologiques anticipatoires. Selon le niveau de satisfaction de ses attentes, l'individu ressent des émotions positives ou négatives (Greiveldinger et al. 2011). Les émotions négatives peuvent se traduire par des comportements de frustration ou de redirection comme des comportements agressifs. Cette notion d'attente chez l'animal, bien caractérisée en psychologie expérimentale, peut être encore difficile à cerner en pratique (Mormede et al. 2018). Ainsi, comment appliquer cette définition du bien-être à nos carnivores domestiques?

\section{ÉVALUER LES ÉMOTIONS}

\section{Définir une émotion}

Une émotion peut être définie comme une réponse affective suite à l'évaluation qu'un individu, humain ou animal, fait de la situation dans laquelle il se trouve (nouveauté, soudaineté, valence, correspondance aux attentes, contrôlabilité...) (Boissy et al. 2007 a,b). Outre la composante évaluative, trois composantes permettent de décrire les émotions : subjective (ce que l'individu ressent), motrice (ce que l'individu montre aux autres : mouvements, expression faciale...) et physiologique (par modification du fonctionnement de l'organisme : sécrétion de cortisol ou modification de la fréquence cardiaque par exemple) (Boissy et al. 2007 a,b). D'après cette définition, une émotion, même si elle est subjective, pourra ainsi être objectivée par des actions motrices (fuite, expression faciale) et par des réactions physiologiques (augmentation de la fréquence cardiaque, de la fréquence respiratoire, de la température interne, des hormones glucocorticoïdes). La réponse de stress (physiologique), plus ou moins importante, est liée à l'intensité de la réponse émotionnelle : plus une émotion est importante, plus la réponse physiologique de stress de l'individu le sera.

\section{Évaluation des émotions positives et négatives}

Ainsi, les émotions sont des réactions instantanées d'un individu, en réponse à un événement. Il est possible d'objectiver et de mesurer des réactions subjectives propres à chaque individu, variant en fonction de son état émotionnel. Un indicateur des émotions réside en l'étude des expressions faciales. Chez l'Homme, six émotions principales sont décrites, à l'origine des autres émotions : la joie, la colère, la tristesse, le dégoût, la peur, la surprise (Ekman \& Friesen 1978 ; Ekman \& Oster 1979 ; Boissy et al. 2007a). Ces expressions faciales sont décrites grâce à la contraction des muscles faciaux, via le FACS (Facial Action Coding System). Chez le chien, notons que les morphotypes très différents de cette espèce peuvent rendre les indicateurs des émotions à partir des expressions faciales difficiles à observer (races brachycéphales, oreilles tombantes, poils recouvrant les yeux, absence de queue...). En particulier, chez les animaux de laboratoire et le cheval, la contraction des muscles faciaux permet d'évaluer la douleur par une grille de " grimace " faciale. Citons par exemple Langford et al. (2010) chez la souris : l'expression de la douleur est liée à la contraction des muscles faciaux, qui entraine un plissement des yeux, un gonflement du museau, les oreilles étant placées en arrière. Chez le cheval, Dalla Costa et al. (2014) proposent également une grille d'expression faciale. De même que chez la souris, la contraction des muscles faciaux entraine : une position des oreilles en arrière, une fermeture de l'œil, une tension musculaire au-dessus de l'œil, des muscles de la mâchoire contractés, un menton plus prononcé, et des naseaux contractés. A notre connaissance, et ceci est surprenant étant donné le développement de la pratique vétérinaire canine et féline, aucune grille de douleur, reposant uniquement sur les expressions faciales et applicable en pratique vétérinaire, n'a été validée pour les animaux de compagnie chiens et chats (Reid et al., 2018). Chez le chien, certaines expressions faciales sont décrites, afin de pouvoir évaluer leurs réactions émotionnelles. Par exemple, Bloom et Friedman (2013) ont pu s'intéresser à sept expressions faciales dans sept contextes différents. Trois photographies d'un chien Berger Belge Malinois ont été retenues par trois experts canins, le chien ayant été confronté aux sept contextes suivants : une expression neutre (contexte neutre : posture assise), de joie (présentation d'une balle au chien), de surprise (boîte qui s'ouvre en face du chien), de tristesse (punition, réprimande), de dégoût (médicament avalé par le chien), de peur (coupe de griffes), et de colère (vocalisation d'un chien connu agressif présentée) (Figure 1). 


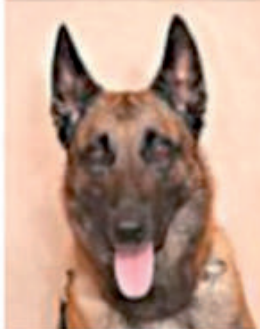

3-Min Sit 1

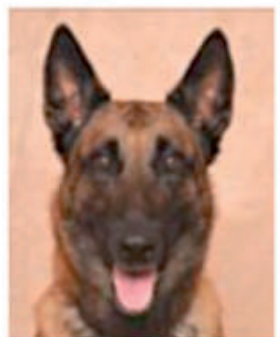

Ball 3

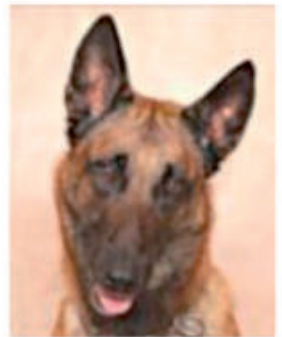

Jack Box 3

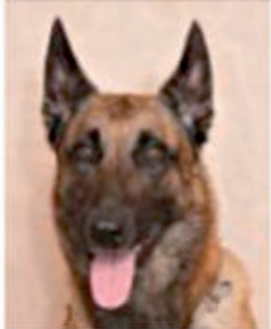

3-Min Sit 6

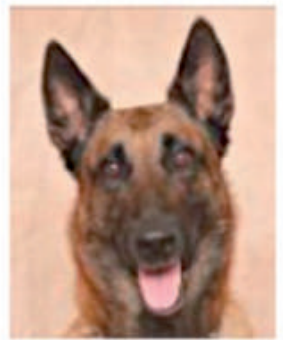

Ball 6

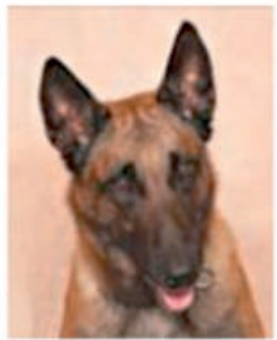

Jack Box 4

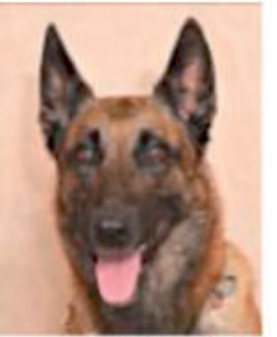

3-Min Sit 8

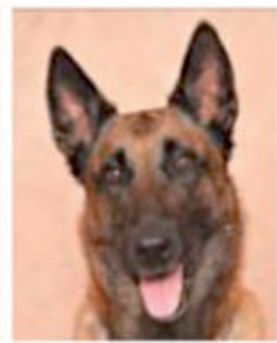

Ball 9

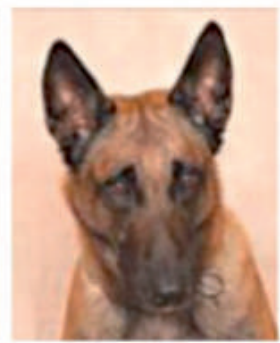

Jack Box 10
Figure 1 : Expressions faciales et émotions chez le chien, d'après Bloom et Friedman (2013)

Ces 21 photos ont été présentées à 50 personnes ( 25 expérimentées et 25 non expérimentées), qui devaient renseigner sur une échelle l'adéquation de la photographie avec les sept émotions proposées. Les résultats montrent que les personnes avaient des difficultés à reconnaître les émotions de tristesse et de peur. Dans $50 \%$ des cas celles expérimentées avaient correctement reconnu le contexte " réprimande " (tristesse), contre 30\% des non-expérimentées. Dans 20\% des cas, les personnes expérimentées avaient correctement reconnu le contexte " coupe de griffe " (peur), contre 30\% des non-expérimentées. Etant donné que plus de $75 \%$ des chiens sont stressés en contexte de consultation vétérinaire (Döring et al. 2009), il est ainsi important que les vétérinaires soient formés afin de reconnaitre les signaux de stress des chiens et chats. En effet, plusieurs publications invitent les praticiens à les détecter pour une meilleure prise en charge (Mariti et al. 2012, 2015). Celle de Herron et Schreyer (2014) en fait la description chez le chat et le chien (Tableau 1). Celle-ci intègre, en plus des expressions faciales, les vocalisations et les postures associées à différentes émotions.

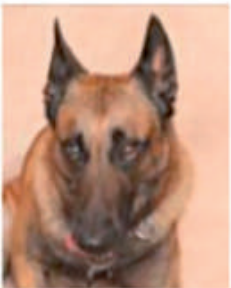

Reprimand 1

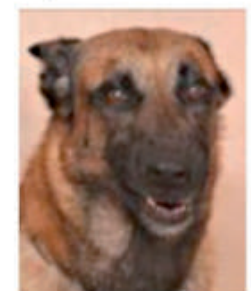

Medicine 4

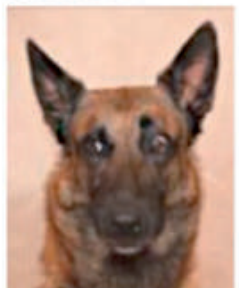

Toe Trim 1

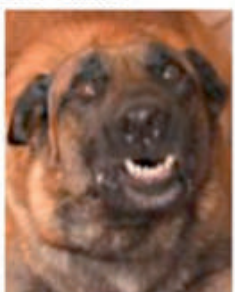

Bad Guy 5

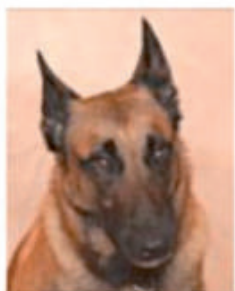

Reprimand 5

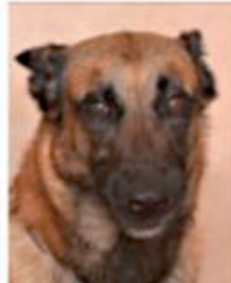

Medicine 5

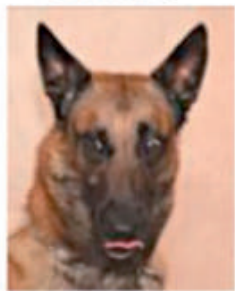

Toe Trim 2

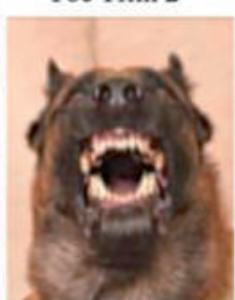

Bad Guy 7

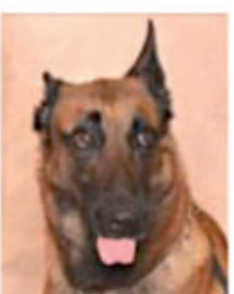

Reprimand 10

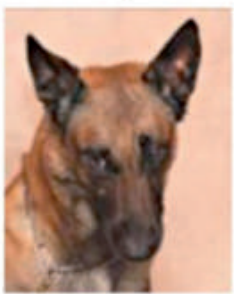

Medicine 9

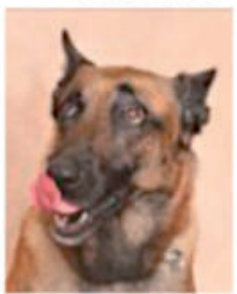

Toe Trim 3

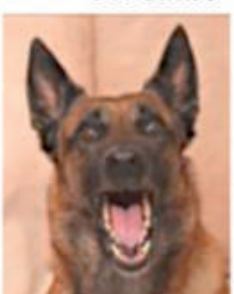

Bad Guy 8
Tableau 1 : Signes d'émotions négatives, peur et stress chez le chien et le chat (d'après Herron $\mathcal{E}$ Schreyer, 2014)

Signes de stress chez le chien Signes de stress chez le chat

Expressions d'une émotion négative (événement perçu comme désagréable, inconfortable) Regard fuyant Léchage de la truffe

Yeux ouverts, blanc de l'œil visible Yeux ouverts

Face tendue, « air fatigué », lèvres pincées

Léchage de la truffe

Déplacement lent (pacing)

Lever de patte (paw lifting)

Bâillement

Gémissement

Ebrouement

Refus de friandise

Expression d'une émotion de peur

Oreilles en arrière $\quad$ Oreilles en arrière

Posture basse, en arrière Posture en arrière, ramassée

Regard détourné Cherche à fuir, éviter la menace

Cherche à fuir, éviter la menace Feulement, crachement

Réaction physiologique de stress, en lien avec une émotion négative intense dans ce contexte \begin{tabular}{ll}
\hline Halètements & Mydriase \\
Tremblements & Haletement
\end{tabular}

Tremblements Halètements

Salivation ou bouche sèche Immobilisation

$\begin{array}{ll}\text { Transpiration } & \text { Salivation ou bouche sèche }\end{array}$

Miction, défécation (si stress très intense) Transpiration

Miction, défécation (si stress très intense) 
Ainsi, la détection des émotions positives et négatives par l'observation des expressions faciales permet d'évaluer le bien-être de l'animal à un moment donné, ceci étant important en pratique vétérinaire afin de favoriser une prise en charge intégrant le ressenti de l'animal au cours des soins. Néanmoins la seule prise en charge " aigüe " de l'animal pendant son passage chez le praticien ne suffit pas à garantir son bien-être. Le vétérinaire doit chercher à détecter les signes d'un mal-être " chronique ", révélateur des conditions de vie de l'animal. Pour cela il faut viser à évaluer son état mental.

\section{ÉVALUER L'ÉTAT MENTAL}

\section{Évaluation de l'état mental par les biais cognitifs}

Afin de pouvoir évaluer les réactions instantanées de l'animal en réponse à une situation donnée, il est possible d'observer ses réponses émotionnelles. Au long cours, la succession d'émotions conduira à déterminer un « état » émotionnel puis mental. L'état émotionnel fait référence à l'état mental ou psychique, modulé en fonction des émotions de l'animal. Cet état représente ainsi plus spécifiquement un état à court terme, intégrant une succession d'émotions (Mormede et al. 2018). Un milieu trop contraignant pourra entrainer une diminution des réactions de l'animal, altérant la prise de décision de celui-ci. En particulier, il est possible d'évaluer la modification des processus cognitifs à travers les tests de " biais cognitif " (Boissy et al. 2007 a,b ; Mendl et al. 2009 ; Henry et al. 2017). Ces tests consistent à apprendre à l'animal une situation favorable (par exemple un seau à gauche récompensé par de la nourriture si l'animal s'en approche) et une situation défavorable (par exemple un seau à droite avec comme conséquence si l'animal s'en approche un mouvement soudain ou un bruit, désagréables). Une situation ambigüe est ensuite présentée à l'animal (par exemple le seau est placé au milieu). On peut ainsi observer si l'animal prend le risque de se déplacer vers le seau (si oui, il est " optimiste ", si non il est "pessimiste "). Les recherches ont montré que des agneaux, confrontés à des stress mineurs répétés, ne se déplaçaient plus vers le seau : on parle de résignation acquise, ou de pessimisme (Boissy et al. 2007b). Ainsi il est possible d'objectiver l'état mental de l'animal à travers les tests de biais de jugement, ou biais cognitif. Même si ces tests ne sont pas réalisables en pratique faute de temps, il est intéressant pour un vétérinaire de pouvoir intégrer la modulation au long cours de l'état émotionnel chez les animaux de compagnie, de loisirs ou de ferme dont il prend soin, en regard des conditions auxquelles il est confronté. Par exemple, la prescription d'une collerette rigide ou de pansements restreignant les mouvements chez les chiens et chats et le comportement de toilettage chez le chat, ou la présence de douleurs chroniques, sont des facteurs qui vont altérer l'état émotionnel des animaux et modifier la perception de leur milieu, de manière négative (possibilités de mouvements restreintes, impossibilité d'exprimer le comportement de toilettage, émotions douloureuses à répétition). Le respect au long terme des émotions des animaux de compagnie, afin de s'assurer de l'absence d'un cumul d'émotions négatives, pourra permettre d'améliorer la prise en charge des soins et l'observance des traitements.

\section{Évaluation par l'utilisation de " scores * de bien-être animal}

Les premières démarches d'évaluation du bien-être à l'échelle européenne ont été développées pour différentes espèces de rente : projets Welfare Quality@ (Blokhuis et al. 2013 : vaches laitières, bovins en engraissement, porcs en engraissement, poules pondeuses, poulets de chair) et AWIN (Animal Welfare Indicators project : cheval, âne, caprins, ovins, dinde). Cette évaluation regroupe 12 critères pour 4 grands principes, permettant d'évaluer le bien-être à travers plusieurs types d'indicateurs : physiologiques, sanitaires, environnementaux, comportementaux. Les critères de bien-être ont été développés à partir des 5 libertés (ou 5 principes ; FAWC, 2009) et incluent des mesures individuelles sur l'animal ainsi qu'une évaluation des ressources disponibles.

Le tableau ci-dessous (Tableau 2) représente les 4 principes et 12 critères pour évaluer le bien-être des vaches laitières (Veissier et al. 2010). Les mesures combinées fournissent un score de bien-être global. Cette grille est considérée comme un référentiel pour la mesure du bien-être des animaux d'élevage. Elle peut être simplifiée pour plus de praticité sur le terrain. La santé est évaluée par trois critères, quatre critères étant liés au comportement. Le critère 12 consiste en une évaluation de l'état émotionnel des animaux.

Tableau 2 : Principes et critères validés par le Welfare Quality (C) (Veissier et al. 2010)

\begin{tabular}{lll}
\hline Principes & Critères \\
\hline Alimentation adatée & 1 & Absence de faim prolongée \\
& 2 & Absence de soif prolongée \\
\hline Logement correct & 3 & Confort autour du repos \\
& 4 & Confort thermique \\
& 5 & Facilité de deplacement \\
\hline Bonne santé & 6 & Absence de blessures \\
& 7 & Absence de maladies \\
& 8 & Absence de douleurs causées par \\
& & les pratiques d'élevage \\
\hline Comportement approprié & 9 & Expression des comportements sociaux \\
& 10 & Expression des autres comportements \\
& 11 Bonne relation Homme-Animal \\
& 12 & Etat émotionnel positif \\
\hline
\end{tabular}

Chez le cheval par exemple, les Animal Welfare Indicators (AWIN) proposent une évaluation du bien-être, toujours avec 4 principes et 12 critères. Cette grille de scores permet en particulier d'évaluer la douleur et le mal-être via la prise en compte des comportements répétitifs et anormaux (stéréotypies). Il convient de noter que le principe "bonne santé " est particulièrement important dans l'évaluation du bien-être, le rôle du vétérinaire est ainsi primordial pour d'une part évaluer le bien-être, mais aussi pour l'améliorer, à travers la prise en compte de la douleur, et son traitement.

Ce type de grille pourrait ainsi être repris afin d'évaluer le bien-être des animaux de compagnie, en intégrant à la fois des critères individus-centrés, et des critères environnement-centrés. Le protocole de Barnard et al. (2014), qui reprend ainsi ce modèle, a été développé pour les chiens en refuge, mais à notre connaissance n'a pas été validé comme l'a pu être le protocole Welfare Quality@. De plus, il n'existe pas à notre connais- 
sance de protocole publié pour les chats en refuge. Une seule étude s'est ainsi attachée à évaluer le bien-être par un système de score pour les animaux de compagnie détenus par un propriétaire, dans un contexte clinique. En effet, un système de score de mal-être a été développé chez des chats de compagnie atteints de prurit cervico-facial (Titeux et al. 2018). Un score maximum de 21 a été déterminé (conditions défavorables), le score minimum étant de 0 (conditions optimales pour le bien-être)

Tableau 3 : Score de malêtre chez les chats de compagnie (Titeux et al. 2018)
(Tableau 3). Les chats atteints de prurit cervico-facial (comportement répétitif) avaient un score supérieur aux chats témoins, révélateur de conditions environnementales défavorables et de perception de l'environnement non optimale. Après enrichissement et modification de l'environnement suite à une consultation en médecine du comportement, les chats ont pu guérir, leur score étant devenu équivalent à celui des chats témoins (Titeux et al. 2018).

\begin{tabular}{|c|c|c|c|c|}
\hline Indicateurs & Score & & Mesures, questions & Significations \\
\hline $\begin{array}{l}\text { 1. Examen médical, } \\
\text { présence de plaie }\end{array}$ & $\begin{array}{l}\text { Non } \\
\text { Oui }\end{array}$ & $\begin{array}{l}0 \\
1\end{array}$ & Examen clinique, présence ou absence de blessures & Présence ou absence de douleur \\
\hline $\begin{array}{l}\text { 2. Adéquation entres les } \\
\text { besoins génétiques du chat } \\
\text { et les conditions de vie }\end{array}$ & $\begin{array}{l}\text { Non } \\
\text { Oui }\end{array}$ & $\begin{array}{l}1 \\
0\end{array}$ & $\begin{array}{l}\text { Est-ce que le chat vient de la campagne ? Est-ce qu'il } \\
\text { a montré des signes de peur ou d'anxiété durant les } \\
\text { premiers mois? }\end{array}$ & $\begin{array}{l}\text { Inadéquation entre génétique et milieu de } \\
\text { vie }\end{array}$ \\
\hline \multicolumn{4}{|c|}{ 3. Accès à la nourriture et à l'eau } & $\begin{array}{l}\text { Investiguer les frustrations par rapport à } \\
\text { l'accès à l'eau et à la nourriture }\end{array}$ \\
\hline Nourriture & $\begin{array}{l}\text { Contrôlé par le chat } \\
\text { Contrôlé en partie par le } \\
\text { propriétaire } \\
\text { Contrôlé par le propriétaire }\end{array}$ & $\begin{array}{l}0 \\
1 \\
2\end{array}$ & $\begin{array}{l}\text { Combien a-t-il de nourriture disponible ? Est-ce qu'il } \\
\text { miaule pour en avoir? }\end{array}$ & \\
\hline Eau & $\begin{array}{l}\text { Contrôlé par le chat } \\
\text { Contrôlé par le propriétaire }\end{array}$ & $\begin{array}{l}0 \\
1\end{array}$ & $\begin{array}{l}\text { L'eau est-elle facilement accessible? Est-ce que le } \\
\text { chat réclame de l'eau du robinet? }\end{array}$ & \\
\hline
\end{tabular}

4. Accès à l'espace (cachette et exploration)

Investiguer les frustrations en rapport avec l'accès à des endroits cachés, des pièces, et des sites d'exploration (fenêtres, balcons, extérieur)

\begin{tabular}{|c|c|c|c|}
\hline $\begin{array}{l}\text { Pièces et endroits pour se } \\
\text { cacher }\end{array}$ & $\begin{array}{l}\text { Contrôlé par le chat } \\
\text { Contrôlé en partie par le } \\
\text { propriétaire } \\
\text { Contrôlé par le propriétaire }\end{array}$ & $\begin{array}{l}1 \\
2\end{array}$ & $\begin{array}{l}\text { Est-ce que le chat a accès à toutes les pièces de la } \\
\text { maison ou de l'appartement ? Est-ce qu'il a des pièces } \\
\text { où se cacher? Est-ce qu'il est autorisé à dormir dans } \\
\text { les placards? Est-ce qu'il demande à aller dans les } \\
\text { placards sans pouvoir le faire? }\end{array}$ \\
\hline Fenêtres & $\begin{array}{l}\text { Contrôlé par le chat } \\
\text { Contrôlé en partie par le } \\
\text { propriétaire } \\
\text { Contrôlé par le propriétaire }\end{array}$ & $\begin{array}{l}1 \\
2\end{array}$ & $\begin{array}{l}\text { Est-ce que le chat à un accès libre aux fenêtres ? Est- } \\
\text { ce que vous acceptez que votre chat demande à aller } \\
\text { sur le rebord de la fenêtre? Quand le temps est } \\
\text { clément est ce que vous acceptez de laisser la fenêtre } \\
\text { ouverte toute la journée ? Est-ce qu'il demande à aller } \\
\text { sur les rebords de fenêtre sans pouvoir le faire? }\end{array}$ \\
\hline Balcon & $\begin{array}{l}\text { Contrôlé par le chat } \\
\text { Contrôlé en partie par le } \\
\text { propriétaire } \\
\text { Contrôlé par le propriétaire }\end{array}$ & $\begin{array}{l}0 \\
1 \\
2\end{array}$ & $\begin{array}{l}\text { Est-ce que le chat est autorisé à passer du temps sur le } \\
\text { balcon ? Est-ce que vous ouvrez le balcon dès que } \\
\text { votre chat demande à y aller? Est-ce qu'il demande à } \\
\text { aller sur le balcon sans pouvoir y accéder? }\end{array}$ \\
\hline Extérieur & $\begin{array}{l}\text { Contrôlé par le chat } \\
\text { Contrôlé en partie par le } \\
\text { propriétaire } \\
\text { Contrôlé par le propriétaire }\end{array}$ & $\begin{array}{l}1 \\
2\end{array}$ & $\begin{array}{l}\text { Si vous avez un jardin, est-ce que le chat est autorisé } \\
\text { à sortir? Est-ce que vous avez une chatière ? Sinon, } \\
\text { est ce que vous ouvrez à votre chat dès qu'il miaule? } \\
\text { Est-ce qu'il demande à aller dans le jardin sans } \\
\text { pouvoir y accéder? }\end{array}$ \\
\hline 5. Relation Homme/chat & $\begin{array}{l}\text { Contrôlé par le chat } \\
\text { Contrôlé en partie par le } \\
\text { propriétaire } \\
\text { Contrôlé par le propriétaire }\end{array}$ & $\begin{array}{l}1 \\
2\end{array}$ & $\begin{array}{l}\text { Est-ce vous portez votre chat? Est-ce que vous } \\
\text { caressez votre chat quand il vous le demande ? } \\
\text { Comment votre chat réagit quand vous le caressez? } \\
\text { Comment réagit votre chat quand il dort sur le canapé } \\
\text { et que vous venez vous asseoir? }\end{array}$ \\
\hline
\end{tabular}




\begin{tabular}{|c|c|c|c|c|}
\hline $\begin{array}{l}\text { 6. Relation avec les autres } \\
\text { chats s'il y a plusieurs chats } \\
\text { dans la maison }\end{array}$ & $\begin{array}{l}\text { Comportement affiliatif } \\
\text { ou pas de chats } \\
\text { En partie agonistique et } \\
\text { en partie affiliative } \\
\text { Agonistique }\end{array}$ & 0 & $\begin{array}{l}\text { Est-ce que vous constatez de l'allogroming / } \\
\text { allorubbing entre vos chats? Est-ce que vos chats } \\
\text { dorment ensemble? Est-ce qu'ils dorment proches } \\
\text { l'un de l'autre? Est-ce qu'ils se battent? Comment } \\
\text { votre chat réagit quand un autre chat entre dans la } \\
\text { pièce alors qu'il est en train de dormir ou quand } \\
\text { l'autre chat veut prendre sa place? }\end{array}$ & Investiguer les relations entre chats \\
\hline $\begin{array}{l}\text { 7. Budget d'activité du chat / } \\
\text { enrichissement / } \\
\text { diversification des activités }\end{array}$ & $\begin{array}{l}\text { Joue fréquemment (plus } \\
\text { d'1 h par jour) } \\
\text { Joue de temps en temps } \\
(<1 \mathrm{~h}) \\
\text { Pas de jouet, pas de jeux }\end{array}$ & $\begin{array}{l}0 \\
1 \\
2\end{array}$ & $\begin{array}{l}\text { Est-ce que vous pouvez me dire à quoi ressemble une } \\
\text { journée typique de votre chat ? Est-ce qu'il y a des } \\
\text { jouets présents dans votre maison (herbe à chat } \\
\text { canne à pêche, balles ...) }\end{array}$ & $\begin{array}{l}\text { Investiguer la qualité et diversité de } \\
\text { l'environnement }\end{array}$ \\
\hline $\begin{array}{l}\text { 8. Adéquation entre le } \\
\text { tempérament du chat et } \\
\text { l'environnement }\end{array}$ & $\begin{array}{l}\text { Adéquation complète } \\
\text { Mitigée } \\
\text { Pas d'adéquation }\end{array}$ & $\begin{array}{l}0 \\
1 \\
2\end{array}$ & $\begin{array}{l}\text { Est-ce que votre chat est timide, peureux, aventurier, } \\
\text { prompt à attaquer, joueur, familier avec l'humain? } \\
\text { Durant l'examen est-ce que le chat est joueur, } \\
\text { explorateur, interagit avec l'humain? }\end{array}$ & Investiguer l'adéquation du milieu de vie \\
\hline $\begin{array}{l}\text { TOTAL } \\
\text { Score minimal = bon score } \\
\text { de bien-être } \\
\text { Score maximal = mauvais } \\
\text { score de bien-être }\end{array}$ & & 21 & & $\begin{array}{l}\text { Pas de problème de bien-être } \\
\text { Problème de bien être important }\end{array}$ \\
\hline
\end{tabular}

\section{Adéquation de l'adaptation de l'animal à son milieu ; quantification de comportements normaux et anormaux}

En parallèle de ces systèmes de scores, il est possible d'observer et de quantifier les comportements normaux (qui doivent être présents dans des proportions adaptées) et anormaux (qui doivent être absents). La démarche, dans l'évaluation du bien-être, de rechercher à la fois les indicateurs positifs et négatifs, est essentielle et adoptée par l'ensemble des acteurs. L'évaluation de l'adéquation entre les besoins de l'animal et le milieu de vie est efficacement schématisée par Fraser et al.
(1997). Ce modèle représente l'adaptation de l'animal à son milieu à l'aide de deux cercles : l'un correspondant aux capacités d'adaptations physiologiques et comportementales de l'individu (dépendant de sa génétique, ses expériences) et l'autre représentant les caractéristiques du milieu de vie. Ainsi, si les deux cercles se recoupent, le niveau de bien-être sera élevé, inversement si les deux cercles n'ont pas de point commun (le milieu ne pouvant plus permettre à l'animal de répondre à ses besoins et de s'adapter), l'animal sera en état de mal-être. Si les deux cercles ne sont pas totalement confondus, et possèdent cependant des points communs, l'adaptation sera possible, alors même que le milieu de vie n'est pas optimal pour l'animal (Figure 2).

\section{Adaptations correspondant à un milieu non confiné qui ne sont plus en lien avec une fonction importante dans le cadre du nouveau milieu}

\section{Défis pour lesquels l'animal ne possède pas les adaptations au nouveau milieu}

\section{cercle A}

\section{Besoins et adaptations de l'animal, acquises avec la sélection (naturelle et artificielle)}

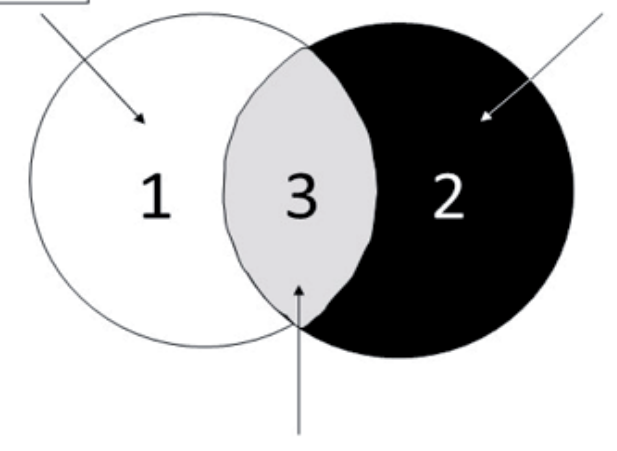

\section{Défis pour lesquels l'animal possède les capacités d'adaptation correspondant au nouveau milieu}

\section{cercle B}

Caractéristiques de

l'environnement

Défis posés à l’animal dans le nouveau milieu (captivité; milieu confiné)

Figure 2 : Adaptations de l'animal à son milieu schématisées par Fraser et al. (1997). 
Cette approche aide à formaliser et expliquer aux propriétaires les différences entre les besoins d'un animal reçu en consultation (chien actif, besoins d'activités mentales, chien familier de l'humain, sociable avec les autres chiens... ou chat prédateur, explorateur, familier ou non de l'humain...) et l'environnement proposé par les propriétaires, afin d'expliquer l'adéquation ou non du milieu. Ceci permet également, pour améliorer le bien-être des carnivores domestiques, de modifier l'environnement d'un individu, ce qui va être l'objectif du vétérinaire dans le cadre d'une consultation, ou de sélectionner pour l'adoption des animaux qui s'adapteront le mieux à leur milieu de vie, plus ou moins contraignant par rapport à leurs besoins.

Comportements normaux : quantification de comportements révélant une adéquation entre les besoins et l'environnement Lorsque le milieu ne correspond pas aux besoins de l'animal, celui-ci ne peut pas réaliser les activités essentielles au cours de la journée dans des proportions adaptées. L'étude des pourcentages d'activités essentielles (alimentation, repos, déplacement, interactions sociales, exploration...) s'appelle l'étude du budget-temps, ou budget d'activité (McFarland, 2009). Chaque espèce possède un budget-temps "type ", même si les individus, différant par leur tempérament, peuvent présenter des variations. Le tempérament correspond à des différences comportementales entre individus, les traits étant constants et consistants dans le temps et les contextes et présents à un âge précoce (Diederich \& Giffroy, 2006). Ainsi, si le milieu correspond aux besoins de l'animal, des indicateurs comportementaux de bien-être pourront être liés à la présence de comportements spécifiques de chaque espèce (déplacements, comportements sociaux...), ainsi qu'à des pourcentages de temps dédiés à des activités essentielles compatibles avec les besoins physiologiques et comportementaux. Le fait que chaque individu soit unique, en particulier en lien avec sa génétique (son tempérament), ses expériences et apprentissages doit être pris en compte pour adapter l'environnement au mieux. Par exemple, certains chats de compagnie ont besoin de sortir (tempérament explorateur, téméraire, prédateur), alors que certains individus pourront tolérer de ne pas avoir accès à l'extérieur. Le modèle de Fraser et al. (1997) peut permettre de visualiser les besoins de chaque animal afin d'adapter au mieux l'environnement.
Comportements anormaux : quantification de comportements anormaux, répétitifs, révélateurs d'une inadaptation de l'animal à son milieu

Lorsque l'animal ne dispose pas des objets adaptés pour satisfaire ses besoins comportementaux, des anomalies peuvent apparaître : reports d'activités vers d'autres objets (activités de substitution), ou activités à vide en l'absence d'objet ou activités répétitives, les stéréotypies (Veissier \& Boissy, 2009). Tout comportement répétitif, stéréotypé, signe ainsi un mal-être, une inadaptation de l'animal à son milieu. Les activités répétitives observées chez les animaux peuvent être : orales (mâchonnements à vide, succion, léchages de l'auge, des murs, des barres des stalles), locomotrices (déambulations, tournis, allers-retours) ou autres (léchages, hyper-toilettage...). Une stéréotypie est définie comme un comportement répétitif, de séquence invariable, et sans fonction apparente (le comportement est déconnecté de sa fonction biologique initiale) (Mason, 1991). Ainsi, ces comportements répétitifs sont révélateurs d'un mal-être. Par exemple chez le chat, le prurit-cervico facial, ou le toilettage excessif (alopécie extensive féline), hors causes dermatologiques, sont apparentés à des comportement répétitifs, signes de mal-être (Titeux et al. 2018). Chez le chien, en particulier les animaux hébergés en chenil, des comportements répétitifs de type tournis, déambulations, léchage des pattes antérieures, aboiements excessifs peuvent être observés. L'enrichissement (ajout d'un ou plusieurs facteurs dans un milieu donné afin d'améliorer le bien-être physique et mental ; Ellis, 2009) diminuera ou supprimera ces comportements répétitifs.

\section{CONCLUSION}

Pour le vétérinaire praticien, le bien-être des carnivores domestiques doit reposer sur deux fondements. Le premier est celui d'une prise en charge respectueuse des émotions de l'individu qui lui est confié, et le deuxième, celui de l'évaluation de l'état mental du chien ou du chat, reflet de ses attentes et de la réponse qu'y apportent ses détenteurs et de l'adéquation de son milieu de vie en lien avec ses besoins. Si les systèmes d'évaluation du bien-être animal ont été développés depuis plusieurs années pour les animaux de production, en particulier à l'échelle européenne, de futures recherches sont nécessaires pour permettre d'évaluer au mieux le bien-être de nos animaux de compagnie.

\section{REMERCIEMENTS}

Les auteurs remercient particulièrement Bertrand Deputte et Jean-Luc Angot de nous avoir fait l'honneur de contribuer à cette séance de l'Académie vétérinaire de France.

\section{BIBLIOGRAPHIE}

- Appleby MC. Letter to the editors. Appl. Anim. Behav. Sci. 1999; 65: 159-162.

- Anses. Bien-être animal : Contexte, définition et évaluation. 2018. https://www.anses.fr/fr/system/files/ SABA2016SA0288.pdf.
- Barnard S, Pedernera C, Velarde A, Villa PD. Welfare assessment protocol for shelter dogs. 2014. http://www.c arodog.eu/wp-content/uploads/2014 102/Shelter-Quality-Protocol-20142.pdf. -Blokhuis H, Miele M, Veissier I, Jones
B. Improving farm animal welfare. Science and society working together: The Welfare Quality approach. Wageningen Academic Publishers, Wageningen, Netherlands. 2013.232 p. - Bloom T \& Friedman H. Classifying 
dogs' (Canis familiaris) facial expressions from photographs. Behav. Processes 2013; 96: 1-10.

- Boissy A, Arnould C, Chaillou E, Colson V, Désiré L, Duvaux-Ponter C et al. Émotions et cognition : stratégie pour répondre à la question de la sensibilité des animaux. In : Numéro spécial, Bien-être animal. INRA Prod. Anim. 2007a ; 20: 17-22.

- Boissy A, Manteuffel G, Jensen MB, Moe RO, Spruijt B, Keeling LJ et al. Assessment of positive emotions in animals to improve their welfare. Physiol. Behav. 2007b; 92: 375-397.

- Dalla Costa E, Minero M, Lebelt D, Stucke D, Canali E, Leach MC. Development of the Horse Grimace Scale (HGS) as a Pain Assessment Tool in Horses Undergoing Routine Castration. Plos One 2014; https://doi.org/ 10.1371/journal.pone.0092281.

- Dawkins M. Behavioural deprivation: a central problem in animal welfare. Appl. Anim. Behav. Sci. 1988; 20: 209-225.

- Diederich C \& Giffroy J-M. Behavioural testing in dogs: A review of methodology in search for standardisation. Appl. Anim. Behav. Sci. 2006; 97: 51-72.

- Döring D, Roscher A, Scheipl F, Küchenhoff $\mathrm{H}$, Erhard MH. Fear-related behaviour of dogs in veterinary practice. Vet. Journal. 2009; 182: 38-43.

- Duncan IJH. Welfare is to do with what animals feel. J. Agric. Environ. Ethics 1993; 6: 8-14.

- Duncan IJH. Poultry welfare: science or subjectivity? Brit. Poultry Sci. 2002; 43: 643-652.

- Ellis S. Environmental enrichment: practical strategies for improving animal welfare. J. Feline Med. Surg. 2009; 11: 901-912.

- Ekman P, Friesen WV. Facial Action Coding System: A technique for the measurement of facial movement. Consulting Psychologists Press, Palo Alto, CA 1978.

- Ekman P, Oster H. Facial expressions of emotion. Annu. Rev. Psychol. 1979, 30, 527-554.

- FAWC. FAWC Report on farm animal welfare in Great Britain: Past, Present and Future. 2009. https://ww w.gov.uk/government/publications/fawc-report-onfarm-animal-welfare-ingreat-britain-past-prese ntand-future.

- Fraser D \& Duncan IJH. Pleasure, pains and animal welfare: toward a natural history of affect. Anim. Welfare 1998; 7: 383-396.

- Fraser D, Weary D-M, Pajor E-A, Milligan B-N. a scientific conception of animal welfare that reflects ethical concerns. Anim. Welfare 1997; 6 : 187-205.

- Greiveldinger L, Veissier I, Boissy A. The ability of lambs to form expectations and the emotional consequences of a discrepancy from their expectations. Psychoneuroendocrinology 2011; 36: 806-815.

- Henry S, Fureix C, Rowberry R, Bateson M, Hausberger M. Do horses with poor welfare show 'pessimistic' cognitive biases? Science of Nature 2017; 104: 8, 15 p.

- Langford DJ, Bailey AL, Chanda ML, Clarke SE, Drummond TE, Echols S et al. Coding of facial expressions of pain in the laboratory mouse. Nat. Methods 2010; 7: 447-449.

- Mariti C, Gazzano A, Moore JL, Baragli P, Chelli L, Sighieri C. Perception of dogs' stress by their owner. J. Vet. Behav. Clin. Appl. Res. 2012; 7: 213-219.

- Mariti C, Raspanti E, Zilocchi M, Carlone B, Gazzano A. The assessment of $\operatorname{dog}$ welfare in the waiting room of a veterinary clinic. Anim. Welfare. 2015;
24: 299-305.

- Mason GJ. Stereotypies: a critical review. Animal Behaviour 1991; 41: 1015-1037.

- McFarland D. Le comportement animal ; Psychobiologie, éthologie et évolution. 2009. Collection : Ouvertures psychologiques. $616 \mathrm{p}$.

- Mendl M, Burman OHP, Parker RMA, Paul ES. Cognitive bias as an indicator of animal emotion and welfare: Emerging evidence and underlying mechanisms. Appl. Anim. Behav. Sci. 2009; 118: 161-181.

- Mormede P, Boisseau-Sowinski L, Chiron J, Diederich C, Eddison J, Guichet J-L et al. Bien-être animal : contexte, définition, évaluation. INRA Prod. Anim. 2018 ; 31 : 145-162.

- Reid J, Nolan AM, Scott EM. Measuring pain in dogs and cats using structured behavioural observation. Vet. J. 2018; 236: 72-79.

- Titeux E, Gilbert C, Briand A, Cochet-Faivre N. From Feline Idiopathic Ulcerative Dermatitis to Feline Behavioral Ulcerative Dermatitis: Grooming Repetitive Behaviors indicators of poor welfare in cats. Front. Vet. Sci. 2018; 5: 81.

- Veissier I \& Boissy A. Evaluation du bien-être des animaux en captivité ou en élevage. In : Alain Boissy (Coordinateur), M.H. Pham-Delègue (Coordinateur), C. Baudoin (Coordinateur), Ethologie appliquée. Comportements animaux et humains, questions de société ; Versailles, FRA, Editions Quae. https://prodinra.inra.fr/record/ 29318. 2009, pp 169-185.

- Veissier I, Botreau R, Perny P. Évaluation multicritère appliquée au bien-être des animaux en ferme ou à l'abattoir : difficultés et solutions du projet Welfare Quality®. INRA Prod. Anim. 2010 ; 23: 269-284. 